\title{
KDM2B overexpression prevents myocardial ischemia-reperfusion injury in rats through regulating inflammatory response via the TLR4/NF-kB p65 axis
}

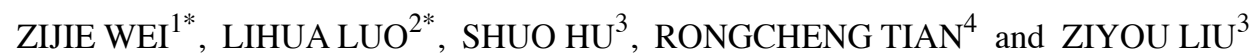 \\ Departments of ${ }^{1}$ Cardiac Intensive Care, ${ }^{2}$ Nephrology, ${ }^{3}$ Cardiac Surgery and ${ }^{4}$ Emergency, \\ The First Affiliated Hospital of Gannan Medical University, Ganzhou, Jiangxi 341000, P.R. China
}

Received August 13, 2021; Accepted October 19, 2021

DOI: $10.3892 /$ etm.2021.11077

\begin{abstract}
Histone modifier lysine-specific demethylase 2B (KDM2B) has been previously reported to activate the inflammatory response by transcription initiation of the $I L-6$ gene. However, the effects of KDM2B on the inflammatory response during myocardial ischemia-reperfusion (I/R) injury and corresponding mechanisms remain poorly understood. The present study aimed to investigate the role and mechanism of KDM2B in myocardial I/R injury. Therefore, a myocardial I/R injury model was established in rats through coronary artery ligation. Adeno-associated virus-encoding KDM2B and small interfering RNA-KDM2B were designed to determine the effects of KDM2B on myocardial I/R injury using $H \& E$ staining and a TUNEL assay in the myocardial tissues. Reverse transcription-quantitative PCR and western blotting were performed to detect the mRNA and protein expression levels of KDM2B, toll-like receptor 4 (TLR4), NF- $\mathrm{B}$ p65 and NOD-, LRR- and pyrin domain-containing protein 3 (NLRP3). ELISA was used to detect the levels of TNF- $\alpha$, IL- 6 and IL- $1 \beta$ in the peripheral blood samples. Pathological analysis demonstrated that the cells in the model group were disordered, with a large area of necrosis and neutrophil infiltration. Knocking down KDM2B expression significantly upregulated the mRNA and protein expression levels of TLR4, NLRP3, NF- $\kappa$ B p65 and the ratio of phosphorylated (p)-p65 to p65. KDM2B knockdown also significantly increased the levels of IL-1 $\beta$, IL- 6 and TNF- $\alpha$ in the peripheral blood, which aggravated myocardial injury and promoted the apoptosis of myocardial cells. However, overexpression of KDM2B downregulated the mRNA and
\end{abstract}

Correspondence to: Dr Ziyou Liu, Department of Cardiac Surgery, The First Affiliated Hospital of Gannan Medical University, 23 Qingnian Road, Ganzhou, Jiangxi 341000, P.R. China

E-mail: ziyoudoc2020@163.com

*Contributed equally

Key words: myocardial ischemia-reperfusion, lysine-specific demethylase $2 \mathrm{~B} /$ toll-like receptor $4 / \mathrm{NF}-\mathrm{kB}$ p65, adenovirus protein expression levels of TLR4, NLRP3, NF- $\kappa$ B P65, the ratio of p-p65 to p65 whilst reducing the levels of IL-1 $\beta$, IL-6 and TNF- $\alpha$ in the peripheral blood, which markedly improved myocardial injury and significantly inhibited the apoptosis of cells in myocardial tissue. In conclusion, the results indicated that overexpression of KDM2B may prevent myocardial I/R injury in rats by reducing the inflammatory response through regulation of the TLR4/NF- $\kappa \mathrm{B}$ p65 axis.

\section{Introduction}

Acute myocardial infarction (AMI) is a disease that has a $30 \%$ mortality rate, where $\sim 50 \%$ mortality occur prior to arrival at the hospital in the United States (1). AMI has been reported to be mainly caused by myocardial ischemia-reperfusion (I/R) injury $(1,2)$. The incidence rate of AMI in the younger population (aged $<45$ years) is rising annually in the United States $(2,3)$. Therefore, strategies to reduce the damage caused by myocardial I/R injury have become a research focus. The pathogenesis of myocardial $\mathrm{I} / \mathrm{R}$ injury refers to a series of complex pathophysiological processes, including the overproduction of reactive oxygen species (4), an inflammatory response (5) and mitochondrial dysfunction (6). Furthermore, it has been demonstrated that the inflammatory response is the main pathological process leading to myocardial injury following reperfusion $(7,8)$.

The histone modifier lysine-specific demethylase 2B (KDM2B) is a member of the JmjC domain-containing histone demethylase family and has been reported to serve a role in lymphomagenesis (9), adipogenesis (10) and the self-renewal of hematopoietic stem cells $(10,11)$. KDM2B is also associated with the occurrence and development of tumors, such as colorectal cancer and T-cell acute lymphoblastic leukemia (12-15). Furthermore, KDM2B is also required for the regulation of choline kinase- $\alpha$ during neuronal differentiation and in maintaining the undifferentiated stage of neuroblasts (16). KDM2B also promotes IL-6 production and the inflammatory response via gene-specific transcription initiation (17). However, to the best of our knowledge, the effects of KDM2B on the inflammatory response in myocardial I/R injury and the corresponding mechanisms are unknown. 
Toll-like receptor 4 (TLR4) is a transmembrane protein, the activation of which promotes $\mathrm{NF}-\kappa \mathrm{B}$ expression and the release of inflammatory factors, such as IL-6, TNF- $\alpha$ and IL-1 $\beta(18,19)$. Therefore, it can be hypothesized that inhibition of the TLR4/NF- $\mathrm{KB}$ signaling pathway could reduce myocardial I/R injury $(20,21)$. Thus, it is of particular significance to identify the role of KDM2B in the inflammatory response in myocardial I/R injury. Therefore, in the present study, a myocardial $\mathrm{I} / \mathrm{R}$ injury rat model was generated to further determine the potential function of KDM2B following overexpression and silencing of KDM2B. The involvement of the inflammatory mechanism was also investigated. The present study provided information on an important therapeutic target for the treatment of myocardial I/R injury.

\section{Materials and methods}

Ethics statement. The protocol for the use of animals in the present study was approved by the Ethics Committee of The First Affiliated Hospital of Gannan Medical University (Ganzhou, China).

Establishment of a myocardial I/R injury model in rats and viral transduction. A total of 30 male Sprague Dawley rats (age, 3 months; weight, $250 \mathrm{~g}$ ) were purchased from Sibeifu (Beijing) Biotechnology Co., Ltd. (license no. scxk 2019-0010; https://www.spf-tsinghua.com/) and housed under consistent conditions at $20^{\circ} \mathrm{C}$ with $30 \%$ humidity in a 12-h light/dark cycle with free access to food and water. The rats were anesthetized by intravenous injection of $1 \%$ pentobarbital sodium $(45 \mathrm{mg} / \mathrm{kg})$. Following anesthesia, the skin was prepared at the neck and cut to separate the subcutaneous fat, after which the trachea was separated. A small hole was inserted into the trachea with a needle 5-8 $\mathrm{mm}$ from the larynx. During surgery, the rats were ventilated with room air using a rodent respirator (Columbus Instruments International) set at 110-120 breaths/min. Left anterior descending coronary artery was ligated with a catheter to block the blood flow. After $50 \mathrm{~min}$ of ligation, the heart was reperfused for $4 \mathrm{~h}$ by removing the catheter. The electrocardiogram (ECG) was monitored. The catheter was removed after 30 min of successful modeling as confirmed by ECG detection based on the reduction of heart rate and elevation of ST segment. The injury of heart tissue was also confirmed by the HE staining, which indicated the heart infarction.

The rats were divided into the following six groups $(n=5$ in each group): A sham group, a model group, an adenoassociated virus (AAV)-small interfering RNA (si/siRNA) KDM2B group, an AAV-siRNA scrambled negative control (NC,pAdEasy-U6-CMV-EGFP) group, an AAV-KDM2B overexpression group and an $\mathrm{AAV}$-overexpression negative control (NC, pAdEasy-EF1-MCS-3FLAG-CMV-EGFP) group. The AAV-encoding siKDM2B (5'-UGGAAGAGGAAGAAGGCA ATTUUGCCUUCUUCCUCUUCCATT-3'), overexpression vectors and corresponding NC (5'-UUCUCCGAACGUGUC ACGUTTACGUGACACGUUCGGAGAATT-3') were provided by Jiangxi Zhonghong Boyuan Biotechnology Co., Ltd. During reperfusion as previously described (22), $4 \mu 1$ adenovirus overexpressing $\mathrm{KDM} 2 \mathrm{~B}\left(1.58 \times 10^{10} \mathrm{PFU} / \mathrm{ml}\right)$ or adenovirus silencing KDM2B $\left(4.0 \times 10^{10} \mathrm{PFU} / \mathrm{ml}\right)$ was locally injected into the margin of the infarcted myocardium. In the sham group, the animals received surgery without ligation and injection of saline. A small hole was also inserted into the trachea as the model group. After the injections, the thoracic cavity was sutured and penicillin $(160,000$ units $/ \mathrm{kg})$ was injected intramuscularly. The rats received $1 \%$ pentobarbital sodium $(120 \mathrm{mg} / \mathrm{kg})$ intraperitoneally $48 \mathrm{~h}$ after reperfusion and decapitated to collect the infarcted heart tissues and $2 \mathrm{ml}$ blood samples, which were stored at $-80^{\circ} \mathrm{C}$ for further use.

$H \& E$ staining. Partial infarcted areas of the heart tissue from three animals were fixed in $4 \%$ paraformaldehyde at $4^{\circ} \mathrm{C}$ overnight. Subsequently, the tissues were washed using water and dehydrated with 70, 80 and $90 \%$ ethanol solutions and added into ethanol absolute and xylene for $15 \mathrm{~min}$. The tissue samples were added to a mixture of xylenes and paraffin for $15 \mathrm{~min}$ and then embedded in paraffin for 50-60 min at room temperature. The tissues were sliced into $10-\mu \mathrm{m}$ sections. The paraffin sections were dried, dewaxed, hydrated and then stained in hematoxylin solution for $3 \mathrm{~min}$ at room temperature, differentiated in ethanol differentiation solution for $15 \mathrm{sec}$, washed with water for $15 \mathrm{sec}$ and stained with eosin for $3 \mathrm{~min}$ at room temperature. The sections were sealed and observed under a light microscope (Olympus Corporation; magnification, x200).

TUNEL assay. Partial infarcted areas of the heart tissue from three animals were fixed in $4 \%$ paraformaldehyde at $4^{\circ} \mathrm{C}$ overnight. The tissues were embedded in paraffin and sectioned into $5-\mu \mathrm{m}$ thickness. The sectioned tissue was placed into a wet box and incubated with $50 \mu \mathrm{g} / \mathrm{ml}$ proteinase $\mathrm{K}$ solution at $37^{\circ} \mathrm{C}$ for $30 \mathrm{~min}$. Each slide was incubated with TUNEL detection solution (cat. no. c1090; Beyotime Institute of Biotechnology) at $37^{\circ} \mathrm{C}$ for $1 \mathrm{~h}$ in the dark and double-stained with $5 \mu \mathrm{g} / \mathrm{ml}$ DAPI for $5 \mathrm{~min}$ at room temperature. Following rinsing with PBS, the sections were incubated with Antifade Mounting Medium solution (including glycerol; cat. no. P0126; Beyotime Institute of Biotechnology) and then observed under a fluorescence microscope (magnification, x200). A researcher (LHL) who was blinded to the groups quantified the number of TUNEL-positive cells in five fields in each section and assessed any histological changes using the Image $\mathrm{J} 2 \mathrm{x}$ v2.0.0 (National Institutes of Health).

Reverse transcription-quantitative PCR (RT-qPCR). Myocardial tissue was ground into powder in liquid nitrogen and was subsequently added to TRIzol reagent (Thermo Fisher Scientific, Inc.). The concentration and purity of RNA [optical density (OD) 260/OD280] were determined using a UV-Vis spectrophotometer. cDNA was synthesized using the HiScript ${ }^{\circledR}$ II Q RT SuperMix for qPCR (cat. no. r223-01; Vazyme Biotech Co., Ltd.) for $50^{\circ} \mathrm{C} 15 \mathrm{~min}$, then $85^{\circ} \mathrm{C}$ for $5 \mathrm{sec}$. The products were used for $\mathrm{qPCR}$, which was performed using the Applied Biosystems StepOnePlus PCR System (Thermo Fisher Scientific, Inc.). Each reaction contained the following: 9.5 $\mu \mathrm{l}$ RNase-free $\mathrm{dH}_{2} \mathrm{O}, 1 \mu \mathrm{l} \mathrm{cDNA}, 1 \mu \mathrm{l}$ forward primer, $1 \mu \mathrm{l}$ reverse primer and $12.5 \mu 12 \mathrm{X}$ SYBR Green PCR Master Mix (cat. no. A4004M; Xiamen Life Internet Technology Co., Ltd.; http://www.lifeint.cn/show_373.htm). The following thermocycling conditions were used for qPCR: Initial denaturation at $95^{\circ} \mathrm{C}$ for $10 \mathrm{~min}$; followed by 40 cycles of denaturation at 
Table I. Sequences of primers used for reverse transcription-quantitative PCR.

\begin{tabular}{|c|c|c|c|}
\hline Gene & Sequence $\left(5^{\prime}-3^{\prime}\right)$ & Primer length, nt & Product length, bp \\
\hline \multirow[t]{2}{*}{ KDM2B } & F: TTCAAACGTCCCCCGGTTC & 19 & \multirow[t]{2}{*}{155} \\
\hline & R: CCAGGACCGCCGCTTT & 16 & \\
\hline \multirow[t]{2}{*}{ TLR4 } & F: CCAGAGCCGTTGGTGTATCT & 20 & \multirow[t]{2}{*}{137} \\
\hline & R: GGCGATACAATTCGACCTGC & 20 & \\
\hline \multirow[t]{2}{*}{ NF-кB p65 } & F: GCAAAAGGACCTACGAGACC & 20 & \multirow[t]{2}{*}{103} \\
\hline & R: CGGGAAGGCACAGCAATA & 18 & \\
\hline \multirow[t]{2}{*}{ NLRP3 } & F: GACCTCAACAGACGCTACACC & 21 & \multirow[t]{2}{*}{102} \\
\hline & R: CCACATCTTAGTCCTGCCAAT & 21 & \\
\hline \multirow[t]{2}{*}{$\beta$-actin } & F: GCCATGTACGTAGCCATCCA & 20 & \multirow[t]{2}{*}{375} \\
\hline & R: GAACCGCTCATTGCCGATAG & 20 & \\
\hline
\end{tabular}

F, forward; KDM2B, lysine-specific demethylase 2B; NLRP3, NOD-, LRR- and pyrin domain-containing protein 3; nt, nucleotide; R, reverse; TLR4, toll-like receptor 4 .

$95^{\circ} \mathrm{C}$ for $10 \mathrm{sec}$, annealing at $58^{\circ} \mathrm{C}$ for $30 \mathrm{sec}$ and extension at $72^{\circ} \mathrm{C}$ for $30 \mathrm{sec}$. The primer sequences were synthesized by General Biosystems (Anhui) Co., Ltd. (Table I). The relative mRNA expression levels of KDM2B, TLR4, NF- $\kappa$ B P65 and NOD-, LRR- and pyrin domain-containing protein 3 (NLRP3) were quantified and normalized to $\beta$-actin using the $2^{-\Delta \Delta \mathrm{Cq}}$ method (23).

Western blotting. Total protein was isolated from myocardial tissue using a TriplePrep isolation kit (cat. no. 28-9425-44; Cytiva). After $30 \mathrm{~min}$ lysis incubation on ice, the homogenates were centrifuged at $11,058 \times \mathrm{g}$ for $15 \mathrm{~min}$ at $4^{\circ} \mathrm{C}$ and the supernatant was carefully extracted to obtain the total protein. Total protein concentration was determined using a BCA kit. The protein was denatured and total protein $(25 \mu \mathrm{g})$ was separated using SDS-PAGE on a $12 \%$ gel. The separated proteins were transferred onto nitrocellulose membranes. Membranes were blocked in 5\% non-fat milk for $1 \mathrm{~h}$ at room temperature and incubated with primary antibodies against KDM2B (cat. no. OM252731; OmnimAbs; 1:1,000; http://www.omnimabs.com/antibody_ FBXL10_antibody_C_term-OM252731.html), TLR4 (cat. no. 19811-1-ap; Proteintech Group, Inc.; 1:1,000), NF-кB p65 (cat. no. 10745-1-ap; Proteintech Group, Inc.; 1:1,000), phosphorylated (p)-p65 (cat. no. AF2006; Affinity Biosciences; 1:1,000), NLRP3 (cat. no. bs-10021R; BIOSS; 1:1,000) and tubulin (cat. no. 10094-1-AP, Proteintech, 1:1,000) overnight at $4^{\circ} \mathrm{C}$. Subsequently, membranes were incubated with the HRP-conjugated anti-mouse IgG secondary antibody (dilution, 1:100; cat. no. ab131368; Abcam) for 1-2 $\mathrm{h}$ at room temperature. Amersham ECL Western Blotting Detection Reagent (cat. no. RPN2134; Cytiva) was added to the membrane. The membrane was imaged using a Bio-Rad Gel Imaging System (Bio-Rad Laboratories, Inc.). Gray density was analyzed using Quantity One Analysis Software version 1.4.6 (Bio-Rad Laboratories, Inc.).

ELISA. ELISAs were used to detect the levels of TNF- $\alpha$ (cat. no. PT516; Beyotime Institute of Biotechnology), IL-6 (cat. no. P I328; Beyotime Institute of Biotechnology) and IL-1 $\beta$ (cat. no. PI303; Beyotime Institute of Biotechnology) in the peripheral blood according to the instructions of the kits.

Statistical analysis. Data are presented as the mean \pm SD. There were five animals in each group. SPSS 21.0 (IBM Corp.) was used to analyze the differences among $\geq$ three groups using one-way ANOVA. In cases where differences were found among groups, Bonferroni's post hoc test was further applied to analyze the difference between two groups. $\mathrm{P}<0.05$ was considered to indicate a statistically significant difference.

\section{Results}

Effects of KDM2B on pathological changes in myocardial I/R injury in rats. The cells in the control group were regularly and orderly arranged, with complete fibers. Cell edema or necrosis was also not observed in the control group. In the model group, siRNA NC group and overexpression NC group, the cell arrangement was disordered, with a large area of necrosis accompanied by neutrophil infiltration. In the si-KDM2B group, the pathological changes were aggravated, whereas the pathological features were improved with no evidence of necrosis or neutrophil infiltration in the KDM2B overexpression group (Fig. 1). These results suggested that KDM2B overexpression may prevent pathological changes in the heart tissue upon I/R injury.

Effects of KDM2B on myocardial apoptosis in myocardial I/R injury in rats. The effect of KDM2B on myocardial apoptosis in rats with myocardial I/R injury was investigated. Compared with the control group, the percentage of apoptotic cells in the heart tissue in the model group was significantly increased $(\mathrm{P}<0.05)$. si-KDM2B further promoted this significant increase compared with that in the siRNA NC group, whereas KDM2B overexpression significantly reduced the extent of apoptosis compared with that in the overexpression NC group $(\mathrm{P}<0.05$; Fig. 2). These results indicated that KDM2B may serve a protective role in the apoptosis of myocardial tissues upon myocardial I/R injury. 


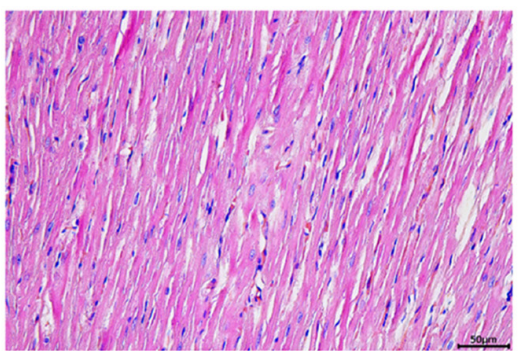

Control

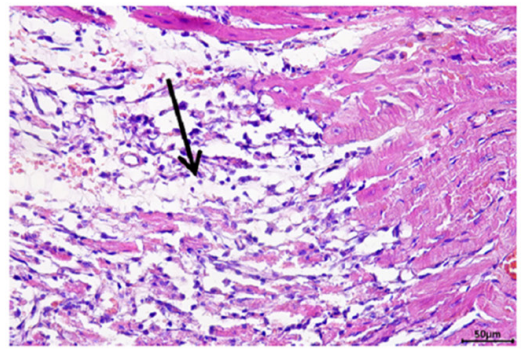

SiRNA NC

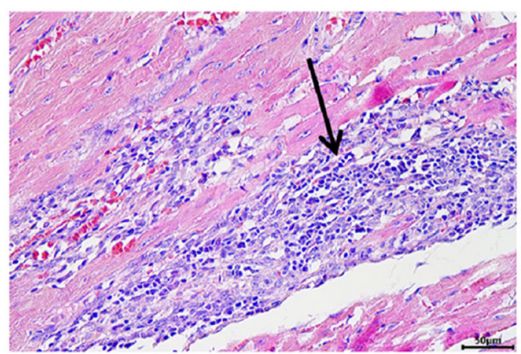

Model

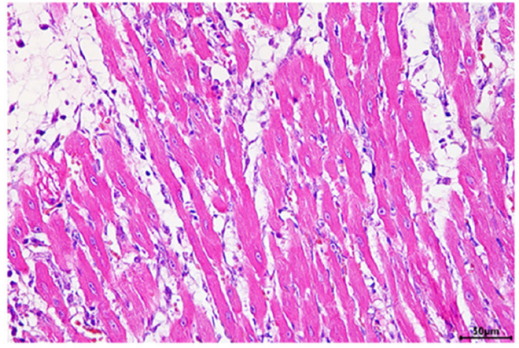

Overexpression

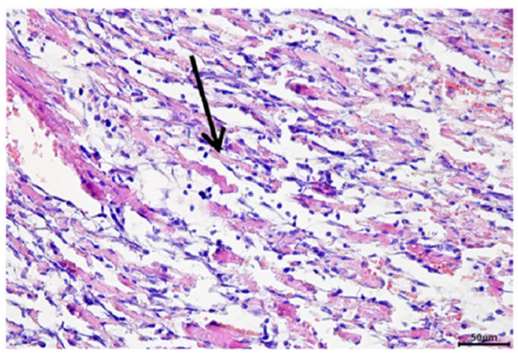

Si-KDM2B

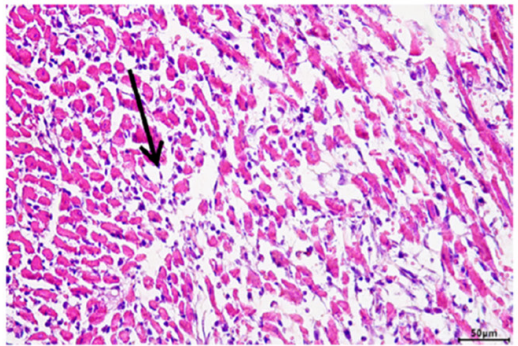

Overexpression NC

Figure 1. Effects of KDM2B on pathological changes in myocardial ischemia-reperfusion injury in rats. In total, six groups, including the sham control group, model group, si-KDM2B group, siRNA NC group, KDM2B overexpression group and overexpression NC group, were included in the present study. H\&E staining was applied to determine the pathological changes. Arrows indicate sites of necrosis and neutrophil infliltration. Scale bars, $50 \mu \mathrm{m}$. AAV, adeno-associated virus; KDM2B, lysine-specific demethylase 2B; NC, negative control; si/siRNA, small interfering RNA; si-KDM2B, AAV-siKDM2B; siRNA NC, AAV-siRNA scrambled negative control.

A
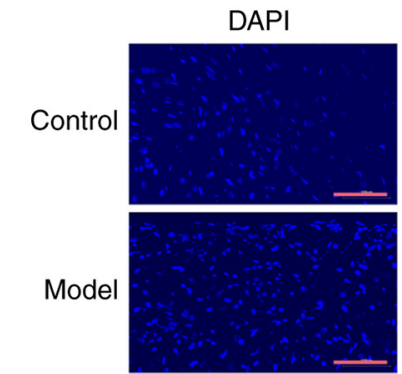

si-KDM2B
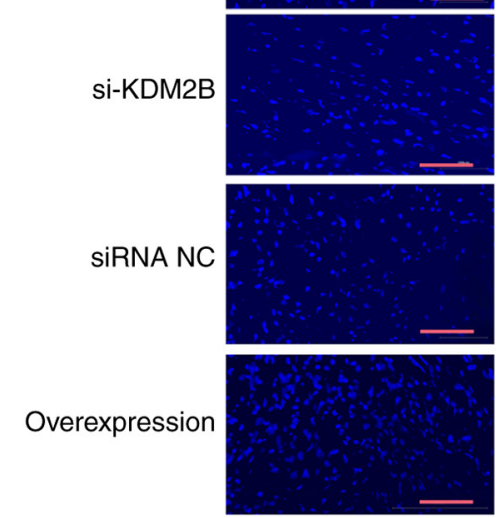

Overexpression NC

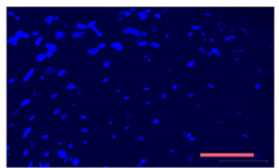

TUNEL
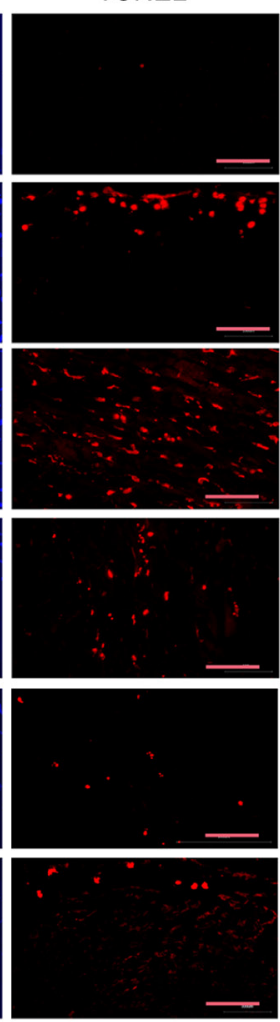
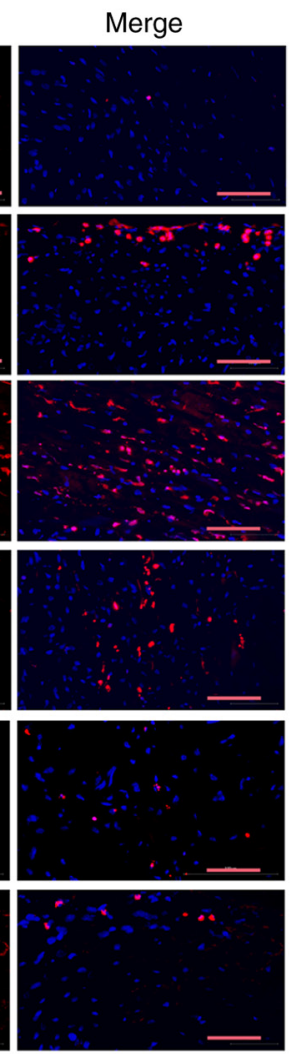

$\mathrm{B}$

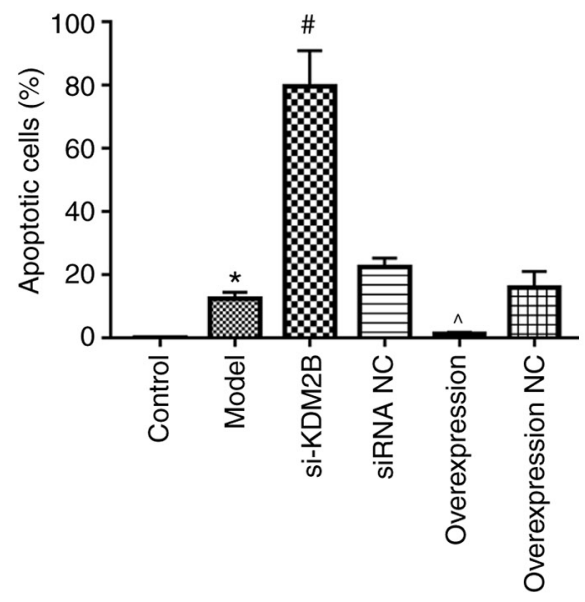

Figure 2. Effects of KDM2B on myocardial apoptosis in myocardial I/R injury in rats. (A) Representative images. Scale bars, $100 \mu \mathrm{m}$. (B) Quantification data. ${ }^{*} \mathrm{P}<0.05$ vs. control; ${ }^{\#} \mathrm{P}<0.05$ vs. siRNA NC; ${ }^{\wedge} \mathrm{P}<0.05$ vs. overexpression $\mathrm{NC}$. AAV, adeno-associated virus; $\mathrm{I} / \mathrm{R}$, ischemia-reperfusion; KDM2B, lysine-specific demethylase 2B; NC, negative control; si/siRNA, small interfering RNA; si-KDM2B, AAV-siKDM2B; siRNA NC, AAV-siRNA scrambled negative control.

Effects of KDM2B on the inflammatory response in myocardial $I / R$ injury in rats. Compared with those of the control group, the levels of IL-1 $\beta$, IL-6 and TNF- $\alpha$ in the peripheral blood of the model group were significantly increased $(\mathrm{P}<0.05$; Fig. 3$)$. Compared with the siRNA NC group, the si-KDM2B group exhibited a further significant 

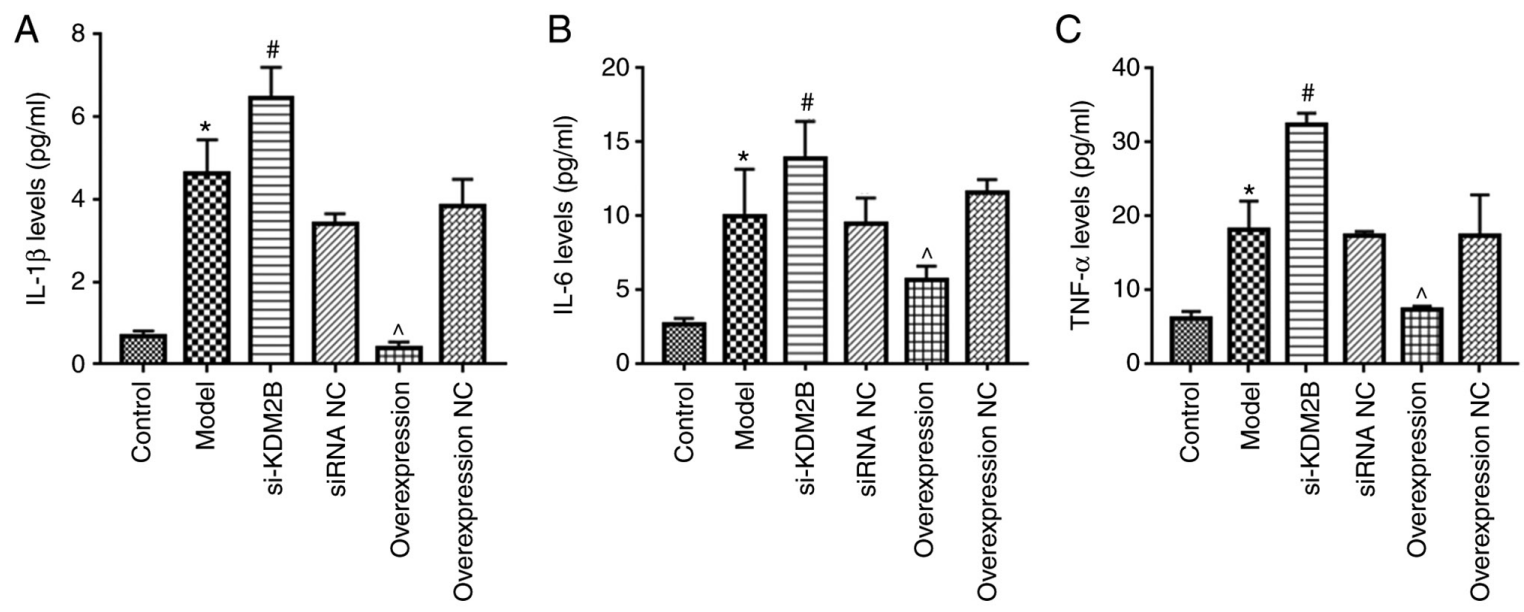

Figure 3. Effects of KDM2B on the inflammatory response in myocardial ischemia-reperfusion injury in rats. Five groups, including the sham control group, model group, si-KDM2B group, siRNA NC group, AAV-KDM2B overexpression group and AAV-overexpression NC group, were included in the present study. The levels of (A) IL-1 $\beta$, (B) IL-6 and (C) TNF- $\alpha$ in peripheral blood were detected via ELISA. " $\mathrm{P}<0.05$ vs. control; ${ }^{*} \mathrm{P}<0.05$ vs. siRNA NC; ${ }^{\wedge} \mathrm{P}<0.05$ vs. overexpression NC. AAV, adeno-associated virus; KDM2B, lysine-specific demethylase 2B; NC, negative control; si/siRNA, small interfering RNA; si-KDM2B, AAV-siKDM2B; siRNA NC, AAV-siRNA scrambled negative control.

increase in the levels of IL-1 $\beta$, IL-6 and TNF- $\alpha(\mathrm{P}<0.05)$, whereas overexpression of KDM2B significantly reduced the levels of IL-1 $\beta$, IL- 6 and TNF- $\alpha$ compared with those in the overexpression NC group $(\mathrm{P}<0.05)$. These results suggested that overexpression of KDM2B may inhibit the inflammatory response in myocardial $\mathrm{I} / \mathrm{R}$ injury in rats.

Effects of KDM2B on TLR4,NF- $\kappa B$ p 65 and NLRP3 expression in myocardial $I / R$ injury in rats. Western blotting demonstrated that KDM2B protein expression was significantly decreased in the model group compared with the control group $(\mathrm{P}<0.05)$. Compared with the corresponding siRNA NC group, si-KDM2B significantly reduced KDM2B protein expression levels further $(\mathrm{P}<0.05)$, whereas KDM2B overexpression significantly promoted KDM2B protein expression compared with that in the overexpression $\mathrm{NC}$ group $(\mathrm{P}<0.05)$.

The protein expression levels of NLRP3, NF- $\mathrm{kB}$ p 65/p-p65 ratio and TLR4 in the model group were significantly higher compared with those of the control group $(\mathrm{P}<0.05)$. Compared with the corresponding siRNA NC group, the protein expression levels of NLRP3, NF- $\mathrm{B}$ p65/p-p65 ratio and TLR4 in the si-KDM2B group were significantly increased further $(\mathrm{P}<0.05)$, whereas the protein expression levels in the overexpression group were significantly reduced compared with those in the overexpression NC group $(\mathrm{P}<0.05$; Fig. 4A and B).

Compared with the control group, the mRNA expression levels of KDM2B were significantly decreased, whereas the mRNA expression levels of NLRP3, NF- $\kappa$ B p65 and TLR4 in the model group were significantly increased $(\mathrm{P}<0.05)$. Compared with the corresponding siRNA NC group, mRNA expression levels of KDM2B in the si-KDM2B group were significantly reduced, and this was accompanied by a significant increase in the expression levels of NLRP3, NF- $\mathrm{kB}$ p65 and TLR4. Furthermore, the mRNA expression levels of KDM2B in the KDM2B overexpression group were significantly increased, whereas the mRNA expression levels of NLRP3, NF- $\mathrm{KB}$ p65 and TLR4 were significantly decreased compared with compared with those in the overexpression $\mathrm{NC}$ group ( $\mathrm{P}<0.05$; Fig. 4C).

\section{Discussion}

In the present study, KDM2B was overexpressed or silenced in the I/R injury rat model. The results demonstrated that reducing KDM2B expression upregulated the protein and mRNA expression levels of TLR4, NLRP3 and NF- $\kappa B$ p65 and promoted the apoptosis of myocardial cells. However, KDM2B overexpression significantly downregulated the mRNA and protein expression levels of TLR4, NLRP3 and NF- $\mathrm{KB}$ p65 and inhibited the apoptosis of myocardial tissues. Therefore, the present study suggest that KDM2B may be a treatment target for myocardial I/R injury.

Histone modification could affect the innate immune response by changing the transcription level of genes (24). KDM2B is a member of the histone lysine demethylase KDM family, which serves important regulatory roles in cell differentiation, development and tumorigenesis $(25,26)$, as well as the inflammatory response $(27,28)$. KDM2B promotes the production of IL-6 and the inflammatory response via brahma-related gene 1-mediated chromatin remodeling (17). Additionally, KDM2B-deficient mice exhibit stronger resistance to endotoxic shock and colitis, a lighter inflammatory phenotype and reduced serum IL-6 production (17). Furthermore, KDM2B expression is reduced in the nasal mucosa of patients with chronic atrophic rhinitis, where reduced KDM2B facilitates the development of nasal mucosa (29). Therefore, KDM2B serves a dual role in the regulation of inflammation in epithelial cells (29). The differential function of KDM2B may be explained by distinctive mechanisms of KDM2B in different cells or diseases. Therefore, the role of KDM2B in inflammation will be a focus of future research. In the present study, KDM2B was silenced or overexpressed in myocardial I/R injury rat models. The results demonstrated that si-KDM2B significantly increased the levels of inflammation in the model rats, which was indicated by the increased levels of IL-1 $1 \beta$, IL- 6 
A

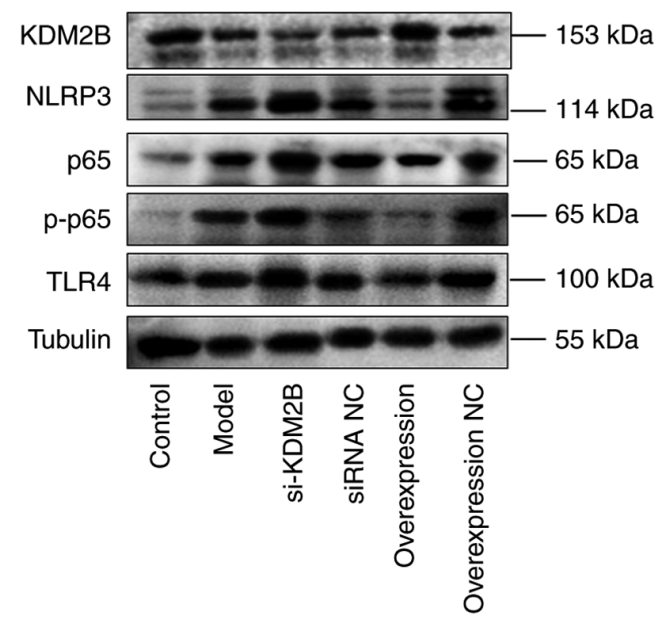

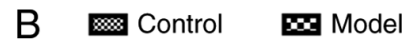

世丑 Overexpression
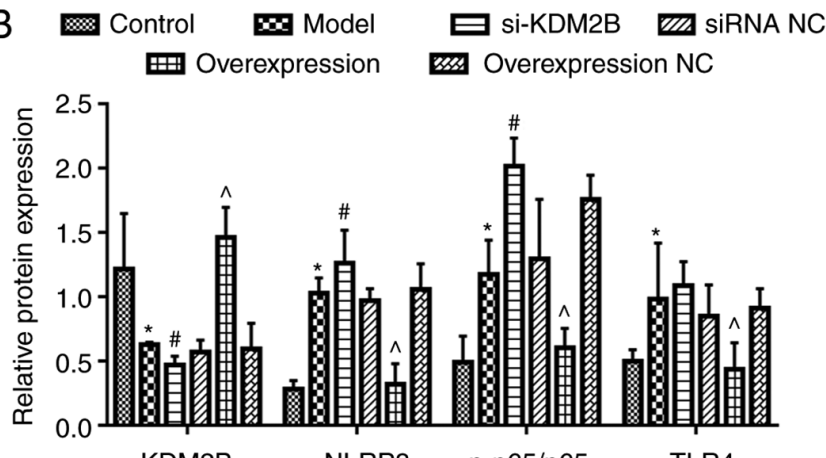

KDM2B
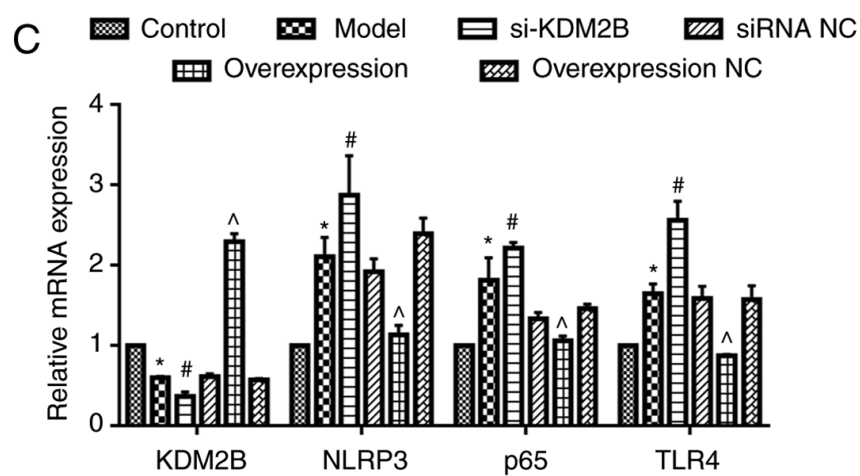

Figure 4. Effects of KDM2B on TLR4, NF- $\mathrm{B}$ p65 and NLRP3 expression levels in myocardial ischemia-reperfusion injury in rats. Five groups, including the sham control group, model group, si-KDM2B group, siRNA NC group, AAV-KDM2B overexpression group and AAV-overexpression NC group, were included in the present study. (A) Western blotting and (B) semi-quantification of protein expression levels. (C) Quantification of mRNA expression levels. ${ }^{*} \mathrm{P}<0.05$ vs. control; ${ }^{\#} \mathrm{P}<0.05$ vs. siRNA NC; ${ }^{\wedge} \mathrm{P}<0.05$ vs. overexpression NC. AAV, adeno-associated virus; KDM2B, lysine-specific demethylase $2 \mathrm{~B} ; \mathrm{NC}$, negative control; NLRP3, NOD-, LRR- and pyrin domain-containing protein 3; p-, phosphorylated; si/siRNA, small interfering RNA; si-KDM2B, AAV-siKDM2B; siRNA NC, AAV-siRNA scrambled negative control; TLR4, toll-like receptor 4.

and TNF- $\alpha$. However, overexpression of KDM2B inhibited the expression of these inflammatory genes. Pathological results also demonstrated that the overexpression of KDM2B improved myocardial tissue injury. The inflammatory response after initial ischemic injury is a key mechanism of secondary degeneration $(30,31)$.

The key role of the NLRP3 inflammasome in regulating the inflammatory response has been demonstrated in numerous studies $(32,33)$. The NLRP3 inflammasome can also increase brain injury and neuroinflammation in ischemic stroke $(34,35)$. In the present study, NLRP3 expression levels were detected and the results demonstrated that myocardial $\mathrm{I} / \mathrm{R}$ injury promoted the mRNA and protein expression of the NLRP3 inflammasome. si-KDM2B further promoted NLRP3 expression. By contrast, overexpression of KDM2B reduced NLRP3 expression. Activation of the NLRP3 inflammasome requires the TLR4/NF- $\kappa B$ signaling pathway to promote the transcription of NLRP3 (36). TLR4 is highly expressed in myocardial cells and vascular endothelial cells, which is closely related to the occurrence and development of cardiovascular disease (37). TLR4 activates the immune and inflammatory response by regulating NF- $\kappa B(32)$ and aggravates myocardial injury $(38,39)$. The results of the present study demonstrated that silencing and overexpression of KDM2B promoted or inhibited the protein and mRNA expression levels of TLR4, NLRP3 and NF- $\mathrm{BB}$ p65, respectively, which is opposite with the trend of KDM2B. These results suggested that KDM2B may regulate the expression levels of proteins associated with the TLR4/NF- $\mathrm{KB}$ signaling pathway.

The present study demonstrated that KDM2B regulated the TLR4/NF- $\kappa \mathrm{B}$ p 65 axis by potentially reducing the inflammatory response, inhibiting cardiomyocyte apoptosis and improving myocardial injury in rats with myocardial I/R injury. However, the regulatory mechanism of KDM2B on the TLR4/NF- $\kappa B$ signaling pathway, whether it depends on the activity of demethylase or directly regulates non-histone proteins, is still unclear. A limitation of the present study was that there are numerous types of cells in myocardial tissues, and thus, the function of KDM2B in specific cell types should be further investigated. Furthermore, the potential of KDM2B as a therapeutic target for myocardial I/R injury will need to be further clarified. In conclusion, overexpression of KDM2B may prevent myocardial $\mathrm{I} / \mathrm{R}$ injury in rats by reducing the inflammatory response via regulation of the KDM2B/TLR4/NF- $\mathrm{kB}$ p65 axis.

\section{Acknowledgements}

Not applicable.

\section{Funding}

The present study was supported by the National Natural Science Foundation of China (grant no. 81960326) and Key R \& D Plan of Jiangxi Provincial Department of Science and Technology (grant no. 20192BBG70039). 


\section{Availability of data and materials}

The datasets used and/or analyzed during the current study are available from the corresponding author on reasonable request.

\section{Authors' contributions}

ZW, LL, SH and RT performed the experiments and analyzed the data. ZW and ZL confirmed the authenticity of all the raw data. ZW and ZL designed the study and wrote the manuscript. All authors read and approved the final manuscript.

\section{Ethics approval and consent to participate}

The present study was approved by the Ethics Committee of the First Affiliated Hospital of Gannan Medical University (approval no. LLSC-2021101202; Ganzhou, China).

\section{Patient consent for publication}

Not applicable.

\section{Competing interests}

The authors declare that they have no competing interests.

\section{References}

1. Anderson JL and Morrow DA: Acute myocardial infarction. N Engl J Med 376: 2053-2064, 2017.

2. Yussman MG, Toyokawa T, Odley A, Lynch RA, Wu G, Colbert MC, Aronow BJ, Lorenz JN and Dorn GW II: Mitochondrial death protein Nix is induced in cardiac hypertrophy and triggers apoptotic cardiomyopathy. Nat Med 8 . 725-730, 2002

3. Lam CK, Zhao W, Cai W, Vafiadaki E, Florea SM, Ren X, Liu Y, Robbins N, Zhang Z, Zhou X, et al: Novel role of HAX-1 in ischemic injury protection involvement of heat shock protein 90 . Circ Res 112: 79-89, 2013.

4. Chouchani ET, Pell VR, Gaude E, Aksentijević D, Sundier SY, Robb EL, Logan A, Nadtochiy SM, Ord ENJ, Smith AC, et al: Ischaemic accumulation of succinate controls reperfusion injury through mitochondrial ROS. Nature 515: 431-435, 2014.

5. Zhang Z, Qin P, Deng Y, Ma Z, Guo H, Guo H, Hou Y, Wang S, Zou W, Sun Y, et al: The novel estrogenic receptor GPR30 alleviates ischemic injury by inhibiting TLR4-mediated microglial inflammation. J Neuroinflammation 15: 206, 2018.

6. Shanmugam K, Ravindran S, Kurian GA and Rajesh M: Fisetin confers cardioprotection against myocardial ischemia reperfusion injury by suppressing mitochondrial oxidative stress and mitochondrial dysfunction and inhibiting glycogen synthase kinase $3 \beta$ activity. Oxid Med Cell Longev 2018: 9173436, 2018.

7. Huang Z, Zhao D, Wang Y, Li X, Li J, Han J, Jiang L, Ai F and Zhou Z: C1q/TNF-related protein 9 decreases cardiomyocyte hypoxia/reoxygenation-induced inflammation by inhibiting the TLR4/MyD88/NF-кB signaling pathway. Exp Ther Med 22: 1139, 2021.

8. Xu XN, Jiang Y, Yan LY, Yin SY, Wang YH, Wang SB, Fang LH and Du GH: Aesculin suppresses the NLRP3 inflammasome-mediated pyroptosis via the Akt/GSK3 $\beta / \mathrm{NF}-\kappa \mathrm{B}$ pathway to mitigate myocardial ischemia/reperfusion injury. Phytomedicine 92: 153687, 2021.

9. Vargas-Ayala RC, Jay A, Manara F, Maroui MA, Hernandez-Vargas H, Diederichs A, Robitaille A, Sirand C, Ceraolo MG, Romero-Medina MC, et al: Interplay between the epigenetic enzyme lysine $(\mathrm{K})$-specific demethylase $2 \mathrm{~B}$ and epstein-barr virus infection. J Virol 93: e00273-19, 2019.
10. Inagaki $\mathrm{T}$, Iwasaki S, Matsumura $\mathrm{Y}$, Kawamura $\mathrm{T}$, Tanaka $\mathrm{T}$, Abe Y, Yamasaki A, Tsurutani Y, Yoshida A, Chikaoka Y, et al: The FBXL10/KDM2B scaffolding protein associates with novel polycomb repressive complex-1 to regulate adipogenesis. J Biol Chem 290: 4163-4177, 2015.

11. Liang G, He J and Zhang Y: Kdm2b promotes induced pluripotent stem cell generation by facilitating gene activation early in reprogramming. Nat Cell Biol 14: 457-466, 2012.

12. Yan M, Yang X, Wang $H$ and Shao Q: The critical role of histone lysine demethylase KDM2B in cancer. Am J Transl Res 10: 2222-2233, 2018

13. Zacharopoulou N, Tsapara A, Kallergi G, Schmid E, Alkahtani S, Alarifi S, Tsichlis PN, Kampranis SC and Stournaras C: The epigenetic factor KDM2B regulates EMT and small GTPases in colon tumor cells. Cell Physiol Biochem 47: 368-377, 2018.

14. Chen L, Fu L, Kong X, Xu J, Wang Z, Ma X, Akiyama Y, Chen Y and Fang J: Jumonji domain-containing protein $2 \mathrm{~B}$ silencing induces DNA damage response via STAT3 pathway in colorectal cancer. Br J Cancer 110: 1014-1026, 2014.

15. Isshiki Y, Nakajima-Takagi Y, Oshima M, Aoyama K, Rizk M, Kurosawa S, Saraya A, Kondo T, Sakaida E, Nakaseko C, et al: $\mathrm{KDM} 2 \mathrm{~B}$ in polycomb repressive complex 1.1 functions as a tumor suppressor in the initiation of T-cell leukemogenesis. Blood Adv 3: 2537-2549, 2019.

16. Domizi P, Malizia F, Chazarreta-Cifre L, Diacovich L and Banchio C: KDM2B regulates choline kinase expression and neuronal differentiation of neuroblastoma cells. PLoS One 14: e0210207, 2019.

17. Zhou Q, Zhang Y, Wang B, Zhou W, Bi Y, Huai W, Chen X, Chen Y, Liu Z, Liu X and Zhan Z: KDM2B promotes IL-6 production and inflammatory responses through Brg1-mediated chromatin remodeling. Cell Mol Immunol 17: 834-842, 2020.

18. Zhou XY, Liu J, Xu ZP, Fu Q, Wang PQ and Zhang $\mathrm{H}$ : Dexmedetomidine inhibits the lipopolysaccharide-stimulated inflammatory response in microglia through the pathway involving TLR4 and NF-кB. Kaohsiung J Med Sci 35: 750-756, 2019.

19. Song Z, Shen F, Zhang Z, Wu S and Zhu G: Calpain inhibition ameliorates depression-like behaviors by reducing inflammation and promoting synaptic protein expression in the hippocampus. Neuropharmacology 174: 108175, 2020.

20. Zhao H, Chen Z, Xie LJ and Liu GF: Suppression of TLR4/NF- $\kappa$ B signaling pathway improves cerebral ischemia-reperfusion injury in rats. Mol Neurobiol 55: 4311-4319, 2018.

21. Arumugam TV, Okun E, Tang SC, Thundyil J, Taylor SM and Woodruff TM: Toll-like receptors in ischemia-reperfusion injury. Shock 32: 4-16, 2009.

22. Zhang Z, Song Z, Shen F, Xie P, Wang J, Zhu AS and Zhu G: Ginsenoside Rg1 prevents PTSD-like behaviors in mice through promoting synaptic proteins, reducing Kir4.1 and TNF- $\alpha$ in the hippocampus. Mol Neurobiol 58: 1550-1563, 2021.

23. Livak KJ and Schmittgen TD: Analysis of relative gene expression data using real-time quantitative PCR and the 2(-Delta Delta C(T)) method. Methods 25: 402-408, 2001.

24. Mehta $\mathrm{S}$ and Jeffrey KL: Beyond receptors and signaling: Epigenetic factors in the regulation of innate immunity. Immunol Cell Biol 93: 233-244, 2015.

25. Chiu WT, Huang YF, Tsai HY, Chen CC, Chang CH Huang SC, Hsu KF and Chou CY: FOXM1 confers to epithelial-mesenchymal transition, stemness and chemoresistance in epithelial ovarian carcinoma cells. Oncotarget 6: 2349-2365, 2015

26. Wang JJ, Dong R, Wang LP, Wang JS, Du J, Wang SL, Shan ZC and Fan ZP: Histone demethylase KDM2B inhibits the chondrogenic differentiation potentials of stem cells from apical papilla. Int J Clin Exp Med 8: 2165-2173, 2015.

27. Souto JA, Sarno F, Nebbioso A, Papulino C, Álvarez R, Lombino J, Perricone U, Padova A, Altucci L and de Lera ÁR: A new family of jumonji $\mathrm{C}$ domain-containing $\mathrm{KDM}$ inhibitors inspired by natural product purpurogallin. Front Chem 8: 312, 2020.

28. Kang MK, Mehrazarin S, Park NH and Wang CY: Epigenetic gene regulation by histone demethylases: Emerging role in oncogenesis and inflammation. Oral Dis 23: 709-720, 2017.

29. Liu CC, Sun C, Zheng X, Zhao MQ, Kong F, Xu FL, Chen XJ, Wang XX, Zhang $M$ and Xia M: Regulation of KDM2B and Brg1 on inflammatory response of nasal mucosa in CRSwNP. Inflammation 42: 1389-1400, 2019.

30. Jin R, Yang G and Li G: Inflammatory mechanisms in ischemic stroke: Role of inflammatory cells. J Leukoc Biol 87: 779-789, 2010. 
31. Hamzei Taj S, Kho W, Aswendt M, Collmann FM, Green C, Adamczak J, Tennstaedt A and Hoehn M: Dynamic modulation of microglia/macrophage polarization by miR-124 after focal cerebral ischemia. J Neuroimmune Pharmacol 11: 733-748, 2016.

32. Shen F, Song Z, Xie P, Li L, Wang B, Peng D and Zhu G: Polygonatum sibiricum polysaccharide prevents depression-like behaviors by reducing oxidative stress, inflammation, and cellular and synaptic damage. J Ethnopharmacol 275: 114164, 2021.

33. Song Z, Bian Z, Zhang Z, Wang X, Zhu A and Zhu G: Astrocytic Kir4.1 regulates NMDAR/calpain signaling axis in lipopolysaccharide-induced depression-like behaviors in mice. Toxicol Appl Pharmacol 429: 115711, 2021.

34. Ma C, Liu S, Zhang S, Xu T, Yu X, Gao Y, Zhai C, Li C, Lei C Fan S, et al: Evidence and perspective for the role of the NLRP3 inflammasome signaling pathway in ischemic stroke and its therapeutic potential (Review). Int J Mol Med 42: 2979-2990, 2018.

35. Sun J, Chi L, He Z, Gao Y, Gao Y, Huang Y and Nan G: NLRP3 inflammasome contributes to neurovascular unit damage in stroke. J Drug Target 27: 866-875, 2019.
36. Kopitar-Jerala N: Innate immune response in brain, NF-kappa B signaling and cystatins. Front Mol Neurosci 8: 73, 2015.

37. Goulopoulou S, McCarthy CG and Webb RC: Toll-like receptors in the vascular system: Sensing the dangers within. Pharmacol Rev 68: 142-167, 2016.

38. Zhang Y, Lu Y, Ma L, Cao X, Xiao J, Chen J, Jiao S, Gao Y, Liu C, Duan Z, et al: Activation of vascular endothelial growth factor receptor-3 in macrophages restrains TLR4-NF- $\kappa B$ signaling and protects against endotoxin shock. Immunity 40 : 501-514, 2014.

39. Li J, Xie C, Zhuang J, Li H, Yao Y, Shao C and Wang H: Resveratrol attenuates inflammation in the rat heart subjected to ischemia-reperfusion: Role of the TLR4/NF- $\kappa \mathrm{B}$ signaling pathway. Mol Med Rep 11: 1120-1126, 2015.

This work is licensed under a Creative Commons Attribution-NonCommercial-NoDerivatives 4.0 International (CC BY-NC-ND 4.0) License. 